\title{
Maternidad tardia: Incidencia, perfiles y discursos
}

\author{
Rosario Sampedro, M. ${ }^{a}$ Victoria Gómez y Mercedes Montero ${ }^{1}$ \\ Departamento de Ciencia Política y Sociología \\ Universidad Carlos III de Madrid \\ sampedro@polsoc.uc3m.es
}

Presentamos en este artículo algunos resultados de una investigación desarrollada entre marzo de 1999 y marzo de 2000 en el Departamento de Ciencia Política y Sociología de la Universidad Carlos III de Madrid, en colaboración con la Dirección General de la Mujer de la Comunidad Autónoma de Madrid. En ella se trataba de analizar la incidencia que la maternidad tardía tiene en esta Comunidad, así como de realizar un esbozo del perfil social de las madres tardías, enmarcando siempre este análisis en una reflexión más amplia sobre el retraso de la maternidad en España y en Europa.

Obviamente la construcción de este objeto de estudio parte de unos presupuestos previos, a saber, a) el carácter cada vez más racional y estratégico del comportamiento reproductivo de las mujeres y de las parejas (los embarazos, en su número y en su calendario, responden cada vez más y de forma mayoritaria a una toma de decisiones «libremente» asumidas, ajenas a presiones institucionales o a la fuerza de la tradición) y b) un elemento fundamental -aunque no el único- a tener en cuenta en el análisis de estas decisiones sobre la reproducción es el género, y en concreto específicas estrategias de género, tendentes a hacer asumibles las particulares exigencias que para la mujer tiene la compatibilización entre vida laboral, carrera profesional y vida familiar (en lo que implica de tiempo y esfuerzo dedicado al cuidado de los niños). Estos presupuestos hacían pertinente una incursión, aunque fuera limitada y exploratoria, en las representacio-

1 Las autoras quieren expresar su agradecimiento a los miembros del Seminario de Demografía del Departamento de Ecología Humana y Población, en la Universidad Complutense de Madrid, por sus sugerentes comentarios. Agradecemos también las aportaciones de los participantes en el Grupo de Trabajo de Sociología de la Población del VII Congreso Español de Sociología, celebrado en Salamanca, en septiembre del 2001. 
nes que sobre la maternidad/paternidad y el ciclo vital aparecen en los discursos de madres y padres tardíos. La indagación cuantitativa sobre la incidencia y el perfil social de la maternidad tardía -basada en una explotación de la Estadística de Partos del Movimiento Natural de la Población de los años 1995, 1996 y 1997 (últimos tres años disponibles en ese momento)- se complementó así con la realización de varias entrevistas en profundidad y grupos de discusión con madres y padres tardíos de la Comunidad Autónoma de Madrid ${ }^{2}$.

\section{LA MATERNIDAD TARDÍA COMO «PROBLEMA». EL PLANTEAMIENTO DE UNA INVESTIGACIÓN}

El género es un elemento fundamental en el planteamiento de nuestra investigación, y ello en varios sentidos. Por un lado como un elemento fundamental de la estructura social, ya que la asignación social a las mujeres de los trabajos relacionados con el cuidado, y la no toma en consideración de esos trabajos reproductivos en la organización espacio-temporal de la vida laboral, en particular, y pública, en general, hace que la mayoría de las mujeres experimenten la vida familiar y la vida profesional y laboral como difícilmente compatibles. Por otro, como elemento ordenador del conocimiento y las representaciones sociales: en esta investigación se adopta explícitamente el punto de vista que sostiene la existencia de sesgos ideológicos relacionados con el género en la práctica científica, que atañen tanto a la construcción de los problemas de investigación, a la elaboración de los conceptos utilizados, a la metodología y a las técnicas de investigación. La demografía, como disciplina que proporciona a la sociología de la población algunos materiales básicos de análisis, no es en absoluto ajena a dichos sesgos, que hemos procurado conocer y evitar en la medida de lo posible.

$\mathrm{El}$ retraso que experimenta la maternidad en las sociedades industriales desarrolladas en los últimos años tiende a presentarse ante la opinión pública como un «problema» al igual que es un «problema», por ejemplo, la maternidad adolescente. Como la maternidad adolescente, la maternidad tardía aparece asociada a la quiebra de determinados modelos familiares, a la pérdida de ciertos valores mora-

${ }^{2}$ Esta investigación de tipo cualitativo se compone de trece entrevistas en profundidad y tres grupos de discusión. Se entrevistó a mujeres que habían sido madres en los últimos cinco años, teniendo treinta o más, algunas asumiendo su maternidad dentro de una relación de pareja, otras formando familias monoparentales, algunas como madres biologicas, y otras en el marco de la adopción. Se entrevistó también a una matrona, con largos años de atención a partos y a los primeros días de la relación madre-hijo, así como a varios padres «tardíos» Los criterios de selección de las mujeres en las entrevistas y en los grupos de discusión han tenido en cuenta los lógicos criterios de homogeneidad-heterogeneidad que toda investigación de este tipo debe contemplar. Las variables, edad a la primera maternidad, nivel socioeconómico y lugar de residencia han sido los cortes utilizados. En general, las personas entrevistadas tienen un nivel económico y cultural medio-alto. El trabajo de campo fue realizado íntegramente en Madrid y su periferia, entre finales del año 1999 y principios del mes de marzo del año 2000 . Los contactos pertinentes para realizar tanto los grupos como las entrevistas se obtuvieron a partir de los datos con que cuenta la Asociación Nacional de Matronas en su sección madrileña. 
les o la disminución de la responsabilidad social de los individuos (sobre todo de las mujeres, en este caso) que les lleva a retrasar el momento de la maternidad hasta los límites de lo biológicamente aceptable. Pero a diferencia de aquélla, la maternidad tardía aparece asociada a la reducción de la fecundidad por debajo de los niveles que aseguran el reemplazo de las generaciones (y a toda una serie de inquietantes ideas, por tanto, acerca de sociedades «envejecidas», o en peligro de desaparición). Y también, como no, a todos los peligros que se intuyen en la manipulación científica de los «límites» que la naturaleza viene imponiendo a los seres humanos desde el principio de los tiempos. Los casos de las madres-abuelas, que han conseguido engendrar a una edad bíblica, ayudadas por modernas técnicas de reproducción asistida, producen no poco asombro e inquietud.

Esta lectura «problemática» de la maternidad tardía no se limita a la opinión pública y publicada, sino que desde ámbitos científicos y académicos el acercamiento a este aspecto del comportamiento reproductivo de las mujeres y de las parejas aparece teñido de matices negativos. En una rápida revisión de los artículos dedicados a este asunto en las principales revistas científicas vemos, por ejemplo, la «maternidad tardía» asociada a «ruptura matrimonial», «infertilidad», «cáncer de mama», «nuevo mundo feliz».....

En este peculiar acercamiento a la maternidad tardía, quizá comprensible desde la biología o la medicina pero no tanto desde las ciencias sociales, podemos ver quizá una manifestación de un fenómeno más amplio que tiene que ver con el tratamiento que desde la demografía y la sociología de la población se hace al comportamiento reproductivo femenino. Como señala Graciela Sarrible (1997), la demografía no es del todo neutral en el tratamiento de los hitos biográficos que ligan a las mujeres a la vida, a la muerte, a la familia o al trabajo. De ahí que sea conveniente adoptar una perspectiva de género, una perspectiva diferente que rompa con determinados supuestos con los que nos encontramos en los análisis demográficos al uso. Uno de los más claros sesgos en este sentido es la obsesión demográfica por la productividad biológica femenina (medida en hijos por mujer) y en último término por la capacidad de reproducción de las poblaciones. La maternidad es sustituida por la fecundidad -y equiparada a ella, aunque tal identidad sea falsa (Sarrible, 1995)- en el interés de la demografía, y todo comportamiento reproductivo que nos aleje del ideal del «reemplazo de las generaciones» es visto como algo «anormal». El retraso de la maternidad se convierte en objeto de interés en la medida en que puede suponer un peligro, esto es, una disminución de la fecundidad final de las generaciones.

En nuestra investigación hemos querido adoptar una perspectiva totalmente distinta. Interesadas en la maternidad de las mujeres, y considerando que la maternidad es algo más amplio y complejo que la mera «productividad biológica», se ha optado por estudiar el perfil social de las madres tardías, a partir de la Estadística de Partos del Movimiento Natural de la Población (única estadística que cuenta mujeres y no nacidos), es decir, nos interesamos por las mujeres que han decidido ser madres, $y$ el parto (definido por el Instituto Nacional de Estadística, como la expulsión de un producto de la concepción viable, con seis o más meses de gestación) es, de los actos que recogen las estadísticas, el que expresa de manera más fidedigna esta decisión. Nuestras mujeres son mujeres que han 
decidido ser madres, y que dan a luz. Vamos a obviar el resultado de esa gestación, de que este acto «produzca» uno o más hijos, o, en el menor de los casos, afortunadamente, se frustre en lo que las frías estadísticas denominan «muertes fetales tardías». Estos principios nos han llevado también a incluir en el análisis cualitativo, entrevistas en profundidad realizadas a madres no biológicas, ya que la adopción, que es en una parte importante maternidad «tardía», es tan maternidad como la biológica.

Definir lo que es la «maternidad tardía» es una tarea más difícil de lo que a primera vista pudiera parecer. Obviamente lo precoz o tardío de un determinado comportamiento depende del contexto social y cultural en el que nos situemos. La medicina nos proporciona una frontera indicativa de lo que debe ser entendido por «tardío» al menos en términos biológicos: madres tardías (o «añosas» como se las denominaba tradicionalmente) son aquellas que se enfrentan a su primera maternidad con 35 años o más. Treinta y cinco años es la edad a partir de la cual las mujeres ven calificado su embarazo como «embarazo de riesgo» y en la que empieza a informarse sistemáticamente a las mujeres de la posibilidad de someterse a pruebas de diagnóstico prenatal, destinadas a detectar posibles enfermedades o malformaciones fetales. Treinta y cinco años es también la edad límite para ser donante de óvulos. Pero las definiciones médicas de lo que es o no tardío no tienen porqué ser un referente para el análisis sociológico, ya que ellas mismas son en gran medida producto de una construcción social.

Una definición sociológica de maternidad tardía debe partir necesariamente de las propias pautas de comportamiento social en este terreno. Lo «tardío» -y somos conscientes de que ese término es un tanto despectivo- debe tener un contenido meramente descriptivo-estadístico, que recoja el cambio social que se está produciendo en este ámbito. En 1998, la edad promedio al nacimiento del primer hijo/a en España era de 28,7 años ${ }^{3}$. España es el tercer país (junto con Irlanda) con la edad promedio al casarse más alta de toda la Unión Europea (solo inferior a la de Dinamarca y Suecia), y el segundo con la edad más alta al tener el primer hijo/a (detrás de los Países Bajos). Aunque la mayoría de las mujeres españolas sigue teniendo su primer hijo/a entre los 25 y los 29 años, el calendario de la fecundidad ha experimentado un considerable retraso en las últimas décadas, que ha quitado a este intervalo de edad su primer puesto en el ranking de la fecundidad. Entre 1971 y 1985 la tasa de fecundidad más alta se daba entre las mujeres de 25 a 29 años, en segundo lugar entre las de 20 a 24 años, y solo en tercer lugar entre las de 30 a 34 años. Aunque la fecundidad disminuyó en todos los grupos de edad, a partir de 1985 la disminución fue mucho más rápida entre las mujeres de 20 a 24 años, de manera que su fecundidad ha sido superada por las de 30 a 34 años desde entonces. La fecundidad de las mujeres de 25 a 29 años ha experimentado también tal disminución que desde 1995 es también inferior a la fecundidad de las mujeres de 30 a 34 años: así pues, el grupo de mujeres de 30 a 34 años, que ha sido tradicionalmente el tercero en fecundidad, ha pasado desde 1995 a ser el de más alta fecundidad

${ }^{3}$ Council of Europe (2000): Recent Demographic Development in Europe, 1999. Strasbourg. 
entre las mujeres españolas, mientras que las mujeres de 35 a 39 años tienen desde 1995 una fecundidad más alta que las de 20 a 24 años, algo que no se había observado nunca en la historia demográfica española (Díez Nicolás, 2001). Puesto que la gran mayoría de las mujeres españolas tienen su primer parto entre los veinticinco y los veintinueve años, aquellas que lo hacen más tarde, tienen una maternidad «tardía»: el grupo de mujeres que deciden ser madres por primera vez entre los 30 y los 34 años están por lo tanto en nuestro punto de mira, aunque en un sentido más estricto (médico) madres tardías serían las que lo hacen con 35 años o más.

Nuestro análisis se aleja de los análisis demográficos clásicos en otro aspecto importante. La demografía analiza problemas que tienen una unidad de tiempo muy larga, que no es el año, es la generación, es la distancia entre padres e hijos, es, en este caso, el comportamiento de distintas generaciones de mujeres a lo largo de su vida fértil. La perspectiva longitudinal es la perspectiva propia y natural de la demografía. La interpretación de indicadores transversales se torna además problemática en situaciones de intenso cambio social. Nuestro análisis es necesariamente transversal y por ello limitado. Analizamos el perfil social de unas mujeres que pertenecen a ciertas generaciones, pero no el comportamiento de esas generaciones. Por supuesto, sabemos que nuestras «madres tardías» son mujeres que han nacido fundamentalmente en la última mitad de la década de los cincuenta y en la primera mitad de la década de los sesenta. Son mujeres que tienen una historia generacional determinada.

La Encuesta de Fecundidad y Familia de 1995 nos puede ayudar a perfilar esta historia generacional. De ella provenían, en el momento de hacer nuestra investigación, los datos más recientes en torno a los comportamientos familiares y reproductivos de las mujeres españolas. Dirigida por Margarita Delgado y Teresa Castro, la EFF-95 presenta algunas innovaciones interesantes respecto a las dos encuestas de fecundidad realizadas anteriormente por el Instituto Nacional de Estadística, en 1977 y en 1985. En ella se entrevistó a varones y se recogieron biografías retrospectivas de la formación de las parejas (incluida la cohabitación), la fecundidad, el uso de anticonceptivos, así como aspectos relacionados con la educación y la formación.

Lo más interesante que aporta la EFF-95, con respecto al estudio del retraso de la maternidad, es la posibilidad de seguir la biografía de diferentes cohortes de mujeres. La comparación entre el calendario que presentan diferentes hitos biográficos en las distintas cohortes (finalización de los estudios, entrada en el mercado de trabajo, abandono del hogar paterno, comienzo de la vida en pareja, llegada del primer hijo...) muestra el constante retraso en todos estos puntos de transición vital. Las cohortes que marcan un cambio de tendencia son las que tienen 30-34 años en 1995 -nacidas en la primera mitad de los sesenta-. Como muy bien señalan las autoras, las mujeres nacidas entre 1960-65 presentan un comportamiento interesante: se observan tendencias divergentes, dependiendo del intervalo de edad, ya que durante la adolescencia presentan una mayor propensión a abandonar la casa paterna que las nacidas diez años antes, pero después de los veinte años ocurre lo contrario. Si observamos el comportamiento reproductivo de esa cohorte, el esquema se repite. 


\section{TABLA 1}

Calendario reproductivo de las mujeres de tres cohortes distintas

\begin{tabular}{|c|c|c|c|}
\hline \multirow[t]{2}{*}{$\begin{array}{l}\text { Porcentaje acumulado de mujeres que } \\
\text { tuvieron el primer nacido vivo a la edad de: }\end{array}$} & $30-34$ & $\begin{array}{c}\text { Edad } \\
35-39 \\
\text { Cohorte de nacimiento }\end{array}$ & $40-44$ \\
\hline & $1960-65$ & $1955-60$ & $1950-55$ \\
\hline 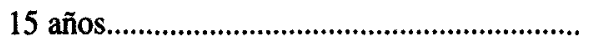 & 0,5 & 0,1 & 0,0 \\
\hline 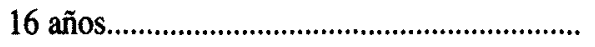 & 1,5 & 0,7 & 0,0 \\
\hline 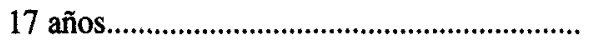 & 3,4 & 1,4 & 1,6 \\
\hline 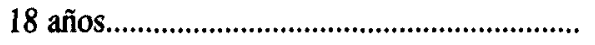 & 6,8 & 3,8 & 3,3 \\
\hline 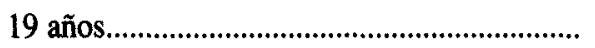 & 11,2 & 7,9 & 4,8 \\
\hline 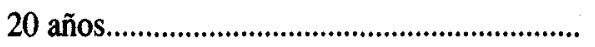 & 16,0 & 15,5 & 8,7 \\
\hline 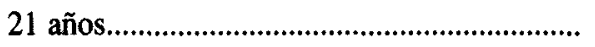 & 21,5 & 23,2 & 15,8 \\
\hline 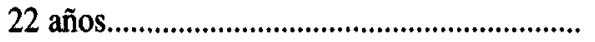 & 21,5 & 23,2 & 15,8 \\
\hline 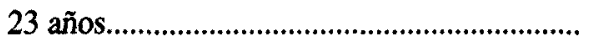 & 31,8 & 44,1 & 34,3 \\
\hline 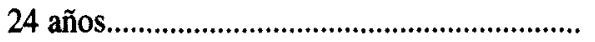 & 38,7 & 50,0 & 47,0 \\
\hline 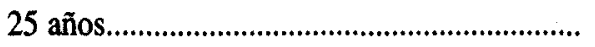 & 46,1 & 58,3 & 58,2 \\
\hline 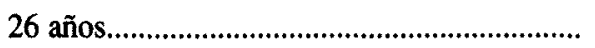 & 52,6 & 65,5 & 65,4 \\
\hline 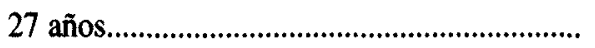 & 58,0 & 71,7 & 72,8 \\
\hline 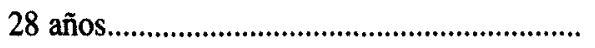 & 63,4 & 75,7 & 77,8 \\
\hline 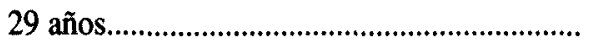 & 70,2 & 79,4 & 80,1 \\
\hline 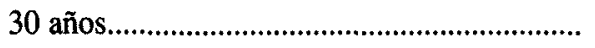 & - & 81,8 & 81,7 \\
\hline
\end{tabular}

Fuente: «Encuesta de Fecundidad y Familia 1995». CIS, 1998, p. 145.

Si comparamos el ritmo de llegada a la primera maternidad de cada cohorte con la precedente, podemos ver que la cohorte nacida entre 1960 y 1965 se escinde entre una minoría de mujeres que tiene una maternidad muy precoz, y una mayoría que la retrasa considerablemente. Comportamientos ambos rupturistas respecto a modelos anteriores. Esta cohorte llega a la adolescencia en plena transición democrática (en 1978, año en que se despenaliza el uso de anticonceptivos tienen entre 13 y 18 años), unos años en los que también sus hermanas mayores (nacidas en la segunda mitad de la década de los cincuenta) están adelantando notablemente su maternidad respecto a cohortes anteriores.

Las mujeres que están bajo nuestro punto de mira, por tanto, llegan a la adolescencia y a la primera juventud en un periodo social y político especialmente convulso y proclive a la adopción de nuevos modelos de conducta, o al menos, a la ruptura con los viejos modelos, un contexto en que las decisiones individuales, el comportamiento estratégico, en definitiva adquiere una mayor relevancia. Esa historia generacional debe ser tenida en cuenta en nuestro análisis.

\section{ESPAÑA EN EUROPA: LA MATERNIDAD TARDÍA EN EL MODELO MEDITERRÁNEO DE FORMACIÓN DE FAMILIAS}

El retraso de la maternidad en el caso español no puede comprenderse sin acudir al marco en el que éste tiene lugar, es decir, sin tener en cuenta la existencia, 
por lo menos a modo de hipótesis, de lo que podríamos llamar un «modelo mediterráneo de formación de las familias».

Uno de los debates que animan el panorama de la demografía y la sociología de la población en los últimos años se centra en la cuestión de si los diferentes modelos familiares apreciables en Europa -en relacion con los distintos niveles de fecundidad, divorcialidad, cohabitación y nacimientos extramatrimoniales- son momentos de un mismo proceso de «desinstitucionalización» de la vida familiar, consolidado ya en el norte y más retrasado, pero en marcha, en el sur, como sugiere Roussel (1992), o bien nos encontramos con modelos diferentes que tienen una base histórica y una lógica social y cultural propia, difícilmente reducibles a esquemas evolucionistas simples (Reher, 1996).

Entre los partidarios de la primera hipótesis se explica la escasa incidencia que en nuestro país tienen las pautas familiares propias de la postmodernidad por el retraso en la emancipación de las generaciones más proclives a adoptarlas, retraso que se atribuye -y aquí nos encontramos de nuevo con posiciones enfrentadas- bien a factores estructurales, «objetivos» o materiales -desempleo, escasez de vivienda, dificultad para compatibilizar vida laboral y familiar, etc(Tobío, 2001b), bien a cambios en los sistemas de valores, en la línea de la tesis del postmaterialismo de Inglehart, combinados con el vigoroso papel asistencial que juega la familia en España (Díez Nicolás, 2001).

Para Roussel, el sur europeo, constituido por España, Italia, Grecia y Portugal, muestra un modelo de familia caracterizado por cifras de fecundidad, divorcialidad, cohabitación y nacimientos no matrimoniales poco elevadas. Este modelo no dejaría de acusar, aunque todavía de forma incipiente, la ola de desinstitucionalización que, según sostiene este autor, se extiende por Europa partiendo de los países escandinavos, desde donde se difunden los nuevos comportamientos, afectando primero a la fecundidad, después a la divorcialidad, posteriormente a las uniones libres no fecundas, y por fin a la cohabitación con niños. Los datos de la EFF-95, a la que nos referíamos anteriormente, parecen apoyar esta tesis: la cohabitación experimenta un aumento considerable entre las cohortes más jovenes, si bien su incidencia es todavía muy pequeña, y otro tanto sucede con los hijos habidos fuera del matrimonio. La cohabitación, no obstante, se concibe como un periodo de prueba previo al matrimonio y es poco fecunda; de hecho, y a diferencia de lo que sucede en otros países las madres solteras españolas son en mayor medida madres solas que madres en uniones de hecho.

Sea por las causas que fuere, España se caracteriza por la fuerza con que la institución familiar preside todavía los comportamientos sociales relativos a la reproducción. En nuestro país el matrimonio sigue constituyendo el requisito previo a la maternidad: de hecho el descenso de la fecundidad en los últimos años es atribuible en gran medida a la disminución y el retraso en la nupcialidad (Requena, 2002), y el aumento de la cohabitación, a diferencia de lo que sucede en otros países europeos, ha sido incapaz de compensar este descenso (Castro, 1999). Al mismo tiempo, la estabilidad laboral y económica de la pareja son requisitos previos al matrimonio, lo que cada vez de forma más 
intensa exige la inserción de las mujeres en el mercado laboral. En España, como señalaba Livi Bacci en una entrevista concedida a un periódico de ámbito nacional ${ }^{4}$, los hijos se hallan al final de un largo proceso. Los jóvenes persiguen proyectos de vida muy claros y muy «acabados» en los que la pareja es para toda la vida -el divorcio es vivido como un verdadero drama personal y familiar-, ha finalizado sus estudios, tiene estabilidad laboral y preferiblemente vivienda en propiedad.

Esta sucesión y consiguiente superación de etapas constituye un elemento básico del escenario cultural en el que las mujeres españolas deciden sobre su maternidad. Pero además, a todas estas circunstancias se une el hecho de que la familia, por encima de su consideración como institución social -y en este punto España acusa plenamente la ola desinstitucionalizadora-, se convierte en lo que Roussel denomina un «pacto privado para la felicidad». La familia española, al tiempo que conserva su capacidad reguladora, vinculada a su carácter de equipo básico de supervivencia frente al papel que los poderes públicos han jugado en otros contextos sociales, está incorporando claramente los valores con que la modernidad tardía impregna las relaciones familiares y de pareja (Meil, 1999). La vida amorosa y familiar ha de proporcionar la máxima satisfacción posible y colmar las aspiraciones personales de los dos miembros de la pareja, siendo la única regla la libertad de cada individuo para decidir en virtud de su deseo de disfrute y autorrealización personal. A pesar de que los comportamientos sociales observados son bastante convencionales, la sociedad española se muestra en todas las encuestas de opinion extremadamente tolerante ante las nuevas formas de convivencia (Tobío, 2001b) ${ }^{5}$. Formas de convivencia que expresan la fuerza de las «relaciones puras» que Giddens describe como el modelo de relación personal propia de nuestro tiempo (Giddens, 1995). Relaciones que se establecen como un ideal, al tiempo que la dinámica de la individualización coloca a hombres y mujeres ante una situación en la que son empujados a buscar una «vida propia» y «plena», a tomar decisiones de forma constante ( $\mathrm{y}$ tomar decisiones supone asumir riesgos) y que lleva, paradojicamente, al tiempo que las relaciones familiares y de pareja se hacen potencialmente más inestables, a aumentar la necesidad de intimidad compartida. El amor se convierte en una religión individual, y se hace tanto más necesario cuando más exigente e imposible (Beck y Beck-Gersheim, 2001).

Una vez encontrada la persona capaz de encarnar el ideal del amor convertido en nueva religión individual, la maternidad y la paternidad compiten además, con otras experiencias vitales consideradas tanto o más enriquecedoras, en un momento en el que se disfruta de independencia económica y social y se dispone de tiempo para viajar, para divertirse, para consumir, etc. La auto-realización personal se impone a otros valores tradicionales como la formación de una familia, dentro del conocido proceso de cambio de los valores materialistas a los post-

4 (El País, 10/11/99).

5 Un buen indicador de esta tolerancia es la cada vez mayor presencia en la publicidad de formas de convivencia familiar no convencionales: jóvenes cohabitantes, familias reconstituidas, relaciones homosexuales, etc... 
materialistas, proceso especialmente intenso en aquellos países, como el nuestro, donde se ha producido un acceso tardío a la sociedad de consumo y un cambio, también tardío, pero muy intenso, en el papel social de la mujer: la importancia de la familia como institución social de asistencia convive con las aspiraciones a vivir «mejor»: con más libertad, autonomía, capacidad de consumo, de auto-expresión y realización personal, con más calidad de vida, en definitiva. (Díez Nicolás, 2001). La familia por tanto, gira en torno a la felicidad de la pareja, y los hijos, cuando llegan, constituyen un elemento más de tal felicidad y realización (Alberdi,1999).

España también comparte con los países mediterráneos otros rasgos que contribuyen igualmente a postergar la maternidad. Nuestro país presenta unos modelos de inserción laboral muy rígidos, que no favorecen en nada la compatibilización de la vida laboral y familiar, con una importancia exigua del trabajo a tiempo parcial en comparación con otros países europeos. España, además, presenta un modelo de régimen de bienestar «conservador», según la clasificación de EspingAndersen (1990), esto es, sustentado fundamentalmente en dos pilares: un concepto tradicional de familia, de inspiración católica, por el cual el núcleo familiar se constituye en una fuente de provisión de cuidados para cada uno de sus miembros; y, por otro lado, del trabajador masculino, perteneciente al núcleo familiar, como el garante, mediante su salario, de la cobertura de las necesidades familiares. La combinación de ambos elementos favorece el que los problemas asociados a la maternidad se consideren exclusivamente como una cuestión familiar o individual y no una responsabilidad social global y que los roles familiares tradicionales tiendan a ser reforzados (Guillén, 1997). En otras palabras, los costes de las cargas familiares son asumidos prácticamente en su totalidad y de forma privada por las familias. Datos de Eurostat correspondientes a 1995, sitúan a España en el último puesto de la Unión Europea en lo que concierne a gasto social destinado a protección familiar: solo un 1,8\% del total del gasto en protección social. La protección social a la familia en España ha sufrido también de forma directa la resaca del largo periodo de dictadura franquista, ya que, asociada a este oscuro periodo de la historia de España, ha sido reducida al mínimo por los sucesivos gobiernos democráticos, si bien en la década de los noventa se produce un cambio de orientación, hacia un reconocimiento de la necesidad de una política familiar (Iglesias de Ussel y Meil, 2001). Aunque de forma reciente se han puesto en marcha algunas medidas tendentes a hacer compatible la vida familiar y laboral, los problemas de la conciliación entre ambas esferas siguen siendo solventados de forma individual y recurriendo, en muchos casos, a la ayuda de la familia (Tobío, 2001a).

La Europa mediterránea se sitúa en este punto muy lejos de los países escandinavos o de los países del Este hasta la caída de los sistemas socialistas, como se puede comprobar en el estudio de Bosvel y Kuijsten (1995) sobre el retraso de la maternidad en cuatro países europeos: Suecia, Hungría Holanda e Italia. La experiencia de los países del Este europeo ponían de manifiesto, hasta el momento de la caída de los regímenes socialistas, la compatibilidad que existe entre actividad y maternidad, incluso a edades muy tempranas. Las sociedades escandinavas, y la sueca sobre todo, han demostrado de forma palpable que la maternidad resulta compatible con altos niveles de participación de la mujer en la 
población activa. Para Roussel, las mujeres escandinavas habrían sido las primeras en adoptar y disfrutar un modelo familiar en el que su igualdad está reconocida y su autonomía garantizada, y de hecho, del entorno de estos países surge la tesis de «a mayor igualdad, niveles de fecundidad más próximos al reemplazo", es decir, que cuando el estatus de la mujer se iguala al del hombre, los niveles de fecundidad tienden a una cierta estabilidad en torno al reemplazo de las generaciones. Esta visión aparece hoy un día en exceso optimista, ya que tras un corto periodo de tiempo, la fecundidad sueca ha vuelto a caer por debajo del nivel de reemplazo, y además el modelo sueco, como el del resto de los países escandinavos se basa en el trabajo a tiempo parcial de las mujeres, con el peligro de reforzar la desigualdad entre los trabajadores «de primera» (hombres) y de «segunda» (mujeres). En cualquier caso, parece obvio que desde las instancias públicas se puede facilitar la conciliación entre vida laboral y vida familiar, y también se puede dificultar, bien por acción o por omisión.

Sin embargo, lo que quizá distinga de forma más evidente a España del resto de los países europeos es el vertiginoso cambio político, social y cultural que nuestro país experimenta en las tres últimas décadas del siglo $\mathrm{XX}$ y con él la drástica transformación de los roles femeninos y el abismo generacional entre madres e hijas, que incluso ha llevado a señalar la existencia de una doble biografía de la mujer en España (Garrido, 1992). Como señalábamos anteriormente son las mujeres nacidas entre 1955 y 1965 , que se encuentran en su adolescencia y primera juventud en plena transición democrática, las primeras que pueden disfrutar de las ventajas de la despenalización de los anticonceptivos en el año 1978, y las que presentarán comportamientos divergentes en el momento elegido para la maternidad, dentro de estas cohortes: una parte de ellas tiene los hijos muy joven, mientras que la otra parte retrasa la maternidad de forma muy notable. Nos encontramos con mujeres que protagonizan una verdadera «revolución reproductiva» (Garrido, 1996), ya que si anteriormente la modificación del calendario reproductivo había girado básicamente en torno al adelanto de la edad a la que se tiene el último hijo, ahora es el retraso en la llegada del primero lo que marca la tendencia (Requena, 1997). Indudablemente se dejará sentir también en nuestro país la influencia de un movimiento feminista muy ligado a la lucha política democrática que en cierta forma desdeñaba la maternidad frente al universo de valores relacionado con la autonomía femenina, y que explica el rechazo de muchas de las mujeres más educadas y comprometidas políticamente a una maternidad vivida como una rémora y asociada a modelos patriarcales del pasado, frente a la exigencia del papel protagonista de la mujer en la esfera pública.

La suma de todos estos elementos contribuye a perfilar el escenario en el que las mujeres españolas toman decisiones sobre su maternidad.

\section{EL PERFIL SOCIAL DE LAS MADRES TARDÍAS EN ESPAÑA}

El Movimiento Natural de la Población (en adelante MNP) recoge anualmente información en torno a ciertas características básicas de las mujeres que dan a 
luz en España: su edad, condición laboral, municipio de residencia, estado civil y años de matrimonio. El MNP ofrece información también acerca de los maridos o parejas de estas mujeres. Es por lo tanto una fuente de información excelente por su periodicidad y extensión, aunque tiene el inconveniente de que el origen de dicha información es la declaración que la propia mujer, su pareja u otro familiar debe realizar en el denominado Boletín Estadístico del Parto, con lo que se hace posibles y reales los errores en los datos, o la no provisión de esos datos en absoluto (como es el caso relativamente frecuente de la información relativa a la condición laboral de las mujeres).

Otro inconveniente grave es que a diferencia de otras fuentes de información estadísticas, como los Censos de Población, los Padrones, o las encuestas realizadas ad hoc, no tenemos las poblaciones de referencia (los «denominadores») que nos permitan evaluar estrictamente la incidencia de determinados fenómenos en determinados colectivos. Cuando nos ha sido posible hemos tratado de paliar esta carencia con datos procedentes de la Encuesta de Población Activa de 1996.

Hemos indagado en el perfil social de las madres tardías españolas a partir de la información contenida en la Estadística de Partos del MNP de los tres últimos años disponibles en el momento de la investigación $(1995,1996,1997)$. La decisión de agregar las mujeres de esos tres años se tomó teniendo en consideración que trabajamos con un colectivo relativamente reducido, y que se nos había pedido un análisis detallado de las madres tardías en la Comunidad Autónoma de Madrid. Se trataba de evitar que para ciertas categorías el número de casos fuera tan reducido que no pudiésemos sacar conclusiones. Hemos trabajado con unas 564.000 mujeres en España y 75.355 en Madrid.

Lo primero que hay que señalar es que la maternidad tardía no tiene demasiada importancia cuantitativa, si por tal consideramos la que se produce por primera vez con 35 años o más. La maternidad tardía estricto senso no llega a un $10 \%$ del total y la maternidad muy tardía (de mujeres mayores de 40 años) está en torno al $1 \%$. Lo que sí tiene importancia es el retraso de la maternidad y la importancia que está tomando la pauta de tener el primer hijo con 30 años o más (entre los 30 y los 34), como podemos ver en las Tablas 2 y 3 . El análisis de la edad de las mujeres a la primera maternidad solo es posible en España desde 1975, año en el que el MNP comienza a facilitar los nacimientos clasificados por rango. Entre 1975 y 1995 la fecundidad en España experimenta una fuerte caída que hace pasar el índice sintético de fecundidad de 2,78 a 1,18 hijos por mujer, y que hace también que los primeros hijos pasen a representar la mitad de todos los nacimientos. El calendario de la fecundidad experimenta un ligero rejuvenecimiento entre 1975 y 1980 , pero a partir de esa fecha muestra un ininterrumpido retraso. Las mujeres de treinta años y más pasan a representar el $35 \%$ de todas las madres que lo son por vez primera. 
TABLA 2

Importancia cuantitativa de la maternidad tardía (porcentaje de primíparas con más de 30, de 35 y de 40 años)

\begin{tabular}{lccc}
\hline $\begin{array}{c}\text { Comunidades } \\
\text { Autónomas }\end{array}$ & Más de 30 años & Más de 35 años & Más de 40 años \\
\hline Andalucía & 28,9 & 5,7 & 0,8 \\
\hline Aragón & 45,4 & 9,3 & 1,0 \\
\hline Asturias & 39,7 & 10,1 & 1,3 \\
\hline Baleares & 33,3 & 7,9 & 1,3 \\
\hline Canarias & 29,9 & 7,3 & 1,2 \\
\hline Cantabria & 41,3 & 9,8 & 1,3 \\
\hline Castilla y León & 44,3 & 9,5 & 1,2 \\
\hline Castilla-La Mancha & 31,0 & 6,5 & 1,1 \\
\hline Cataluña & 40,0 & 8,7 & 1,1 \\
\hline C. Valenciana & 35,3 & 7,1 & 1,1 \\
\hline Extremadura & 27,7 & 5,7 & 0,8 \\
\hline Galicia & 32,2 & 8,0 & 1,2 \\
\hline C. de Madrid & 45,8 & 9,7 & 1,2 \\
\hline Murcia & 28,1 & 5,8 & 0,9 \\
\hline Navarra & 49,5 & 10,3 & 1,2 \\
\hline País Vasco & 54,2 & 10,8 & 1,0 \\
\hline La Rioja & 43,0 & 9,1 & 0,9 \\
\hline Ceuta y Melilla & 28,0 & 8,7 & 1,4 \\
\hline ESPAÑA & 37,2 & 8,0 & 1,1 \\
\hline
\end{tabular}

Fuente: Movimiento Natural de la Población, INE, Media de los años 1995, 1996, 1997. Elaboración propia. 
TABLA 3

Primeros nacimientos, según edad de la madre (en porcentaje sobre el total)

\begin{tabular}{ccccccccc}
\hline Años & $\begin{array}{c}\text { Menos } \\
\text { de 15 }\end{array}$ & $\mathbf{1 5 - 1 9}$ & $\mathbf{2 0 - 2 4}$ & $\mathbf{2 5 - 2 9}$ & $\mathbf{3 0 - 3 4}$ & $\mathbf{3 5 - 3 9}$ & $\mathbf{4 0} \mathbf{y}$ más & Total \\
\hline 1975 & 0,1 & 10,4 & 44,7 & 33,5 & 8,1 & 2,3 & 0,1 & 100 \\
\hline 1976 & 0,1 & 11,9 & 47,0 & 30,6 & 7,5 & 2,0 & 0,8 & 100 \\
\hline 1977 & 0,1 & 12,7 & 47,5 & 29,5 & 7,5 & 1,9 & 0,7 & 100 \\
\hline 1978 & 0,1 & 13,7 & 46,9 & 29,0 & 7,6 & 2,0 & 0,7 & 100 \\
\hline 1979 & 0,1 & 14,5 & 47,2 & 27,6 & 7,6 & 2,2 & 0,7 & 100 \\
\hline 1980 & 0,2 & 14,2 & 45,7 & 28,2 & 8,1 & 2,6 & 1,0 & 100 \\
\hline 1981 & 0,2 & 13,4 & 44,8 & 29,2 & 8,5 & 2,8 & 1,0 & 100 \\
\hline 1982 & 0,2 & 13,0 & 43,3 & 30,4 & 8,9 & 3,0 & 1,1 & 100 \\
\hline 1983 & 0,2 & 13,1 & 41,4 & 32,0 & 9,2 & 3,1 & 1,1 & 100 \\
\hline 1984 & 0,1 & 12,6 & 39,8 & 33,6 & 9,6 & 3,2 & 1,1 & 100 \\
\hline 1985 & 0,1 & 12,2 & 37,7 & 35,6 & 10,0 & 3,2 & 1,1 & 100 \\
\hline 1986 & 0,1 & 11,7 & 36,5 & 37,0 & 10,6 & 3,1 & 1,1 & 100 \\
\hline 1987 & 0,1 & 11,0 & 34,3 & 38,6 & 11,7 & 3,3 & 1,1 & 100 \\
\hline 1988 & 0,1 & 10,6 & 32,8 & 39,6 & 12,7 & 3,2 & 1,0 & 100 \\
\hline 1989 & 0,1 & 9,3 & 30,7 & 41,0 & 14,4 & 3,5 & 1,0 & 100 \\
\hline 1990 & 0,1 & 8,5 & 28,6 & 42,3 & 15,8 & 3,7 & 1,0 & 100 \\
\hline 1991 & 0,1 & 7,8 & 26,0 & 42,8 & 18,1 & 4,1 & 1,1 & 100 \\
\hline 1992 & 0,1 & 7,1 & 23,7 & 43,5 & 20,0 & 4,7 & 1,1 & 100 \\
\hline 1993 & 0,1 & 6,5 & 21,4 & 43,6 & 22,2 & 5,2 & 1,1 & 100 \\
\hline 1994 & 0,1 & 6,1 & 19,5 & 42,7 & 24,6 & 5,9 & 1,2 & 100 \\
\hline 1995 & 0,1 & 5,7 & 17,7 & 41,7 & 27,0 & 6,5 & 1,3 & 100 \\
\hline
\end{tabular}

Fuente: Movimiento Natural de la Población, años correspondientes. Elaboración propia.

Si nos fijamos en tres aspectos clave del perfil social de las madres tardías (condición laboral, estado civil y tiempo transcurrido desde el momento del matrimonio y el primer parto, en el caso de las mujeres casadas) podemos tener una primera imagen interesante de las madres tardías españolas. Como era esperable existe una asociación clara entre la situación laboral de las mujeres y la edad al primer parto. Aquellas mujeres que desarrollan su trabajo en el ámbito familiar, tienen las medias de edad al primer parto más bajas (amas de casa y agricultoras: 26,34 y 26,36). A medida que se incrementa la cualificación laboral aumentan las medias: las trabajadoras menos cualificadas en torno a los 28 años y las trabajadoras cualificadas por encima de los 29 (profesionales y técnicas 30,69; directivas 29,56; y administrativas 29,51). 
TABLA 4

Media de edad al primer parto según situación laboral. Datos nacionales

\begin{tabular}{lrrc}
\hline $\begin{array}{c}\text { Situación laboral de } \\
\text { la mujer }\end{array}$ & Número & $\%$ & $\begin{array}{c}\text { Media de edad } \\
\text { al primer parto }\end{array}$ \\
\hline Profesional, Técnica & 88916 & 15,8 & 30,69 \\
\hline Directiva & 3250 & 0,6 & 29,91 \\
\hline Administrativa & 85583 & 15,2 & 29,56 \\
\hline Comerciante & 31223 & 5,5 & 28,20 \\
\hline Servicios & 48366 & 8,6 & 28,19 \\
\hline Agricultora & 4082 & 0,7 & 26,36 \\
\hline Obrera & 24608 & 4,4 & 28,04 \\
\hline Militar & 362 & 0,1 & 26,56 \\
\hline Estudiante & 8900 & 1,6 & 22,30 \\
\hline Ama de casa & 179960 & 31,9 & 26,34 \\
\hline Jubilada & 177 & 0,0 & 30,50 \\
\hline No clasificada & 88432 & 15,7 & 27,14 \\
\hline Total & 563859 & 100,0 & 27,93 \\
\hline
\end{tabular}

Fuente: Movimiento Natural de la Población. Media de los años 1995, 1996 y 1997. Elaboración propia.

Si consideramos tres grandes categorías laborales (trabajadoras cualificadas, no cualificadas y amas de casa) podemos ver también que las madres tardías son con mucha mayor frecuencia trabajadoras con una alta cualificación. Es interesante comprobar que las mujeres en la treintena presentan un perfil laboral muy similar, mientras que aquellas que se encuentran en los cuarenta tienen mayores niveles de domesticidad (Tabla 5).

TABLA 5

Situación laboral de las mujeres primíparas según edad. Datos nacionales

\begin{tabular}{lrrrrrr}
\hline Situación laboral de la mujer & $\begin{array}{c}\text { Menor } \\
\text { de 20 }\end{array}$ & $\mathbf{2 0 - 2 4}$ & $\mathbf{2 5 - 2 9}$ & $\mathbf{3 0 - 3 4}$ & $\mathbf{3 5 - 3 9}$ & $\begin{array}{c}\text { Mayor } \\
\text { de 40 }\end{array}$ \\
\hline $\begin{array}{l}\text { Profesionales, directivas y } \\
\text { administrativas }\end{array}$ & 1,3 & 8,8 & 31,0 & 47,4 & 46,3 & 35,6 \\
\hline Empleadas y obreras & 7,3 & 17,5 & 21,3 & 17,8 & 16,5 & 15,4 \\
\hline Amas de casa & 54,6 & 49,3 & 31,9 & 20,5 & 21,0 & 30,0 \\
\hline Resto & 36,6 & 24,6 & 15,9 & 14,1 & 16,1 & 19,1 \\
\hline Total & 100,0 & 100,0 & 100,0 & 100,0 & 100,0 & 100,0 \\
\hline
\end{tabular}

Fuente: Movimiento Natural de la Población. Media de los años 1995, 1996 y 1997. Elaboración propia. 
Obviamente ello no quiere decir que las mujeres profesionales y técnicas sean fundamentalmente madres tardías o que las amas de casa tengan más probabilidad de ser madres primerizas con más de cuarenta años que con 35-39, como podemos ver si tenemos en cuenta el número total de mujeres en cada categoría de edad y condición laboral, que nos proporciona la EPA (Tabla 6).

\section{TABLA 6}

Porcentaje de primíparas sobre total de mujeres en cada categoría laboral y grupo de edad

\begin{tabular}{lrrrrrr}
\hline Situacion laboral de la mujer & $\begin{array}{c}\text { Menor } \\
\text { de 20 }\end{array}$ & $\mathbf{2 0 - 2 4}$ & $\mathbf{2 5 - 2 9}$ & $\mathbf{3 0 - 3 4}$ & $\mathbf{3 5 - 3 9}$ & $\begin{array}{c}\text { Mayor } \\
\text { de 40 }\end{array}$ \\
\hline $\begin{array}{l}\text { Profesionales, directivas y } \\
\text { administrativas }\end{array}$ & 1,8 & 3,4 & 16,7 & 19,5 & 4,8 & 0,4 \\
\hline Empleadas y obreras & 1,8 & 3,6 & 12,7 & 8,2 & 1,7 & 0,1 \\
\hline Amas de casa & 27,2 & 45,2 & 31,0 & 7,6 & 1,6 & 0,1 \\
\hline Resto & 1,0 & 2,6 & 11,9 & 13,8 & 3,9 & 0,5 \\
\hline
\end{tabular}

Fuente: Elaboración propia con datos del Movimiento Natural de la Población (media de los años 1995, 1996 y 1997) y Encuesta de la Población Activa 1996.

Lo más destacable si consideramos el estado civil es la gran incidencia que tiene la maternidad fuera del matrimonio entre las primíparas más jóvenes y las más mayores, lo que puede expresar, por un lado, la «excepcionalidad social» de esos dos tipos de maternidad, y por otro lado la adopción de nuevos modelos de familia entre las madres tardías (Tabla 7). Si tenemos en cuenta, sin embargo, el número total de mujeres en cada categoría de edad y estado civil vemos que la maternidad fuera del matrimonio es algo que incide más en las mujeres de 30 a 39 años (Tabla 8).

TABLA 7

Estado civil de las mujeres primíparas según edad. Datos nacionales

\begin{tabular}{lrrrrrr}
\hline Estado Civil & $\begin{array}{c}\text { Menor } \\
\text { de 20 }\end{array}$ & $\mathbf{2 0 - 2 4}$ & $\mathbf{2 5 - 2 9}$ & $\mathbf{3 0 - 3 4}$ & $\mathbf{3 5 - 3 9}$ & $\begin{array}{c}\text { Mayor } \\
\text { de 40 }\end{array}$ \\
\hline Casadas primeras nupcias & 44,0 & 74,7 & 90,8 & 89,1 & 77,9 & 71,2 \\
\hline Casadas segundas nupcias & 0,3 & 0,4 & 0,5 & 1,1 & 2,7 & 3,5 \\
\hline No casadas & 55,6 & 24,8 & 8,7 & 9,8 & 19,4 & 25,3 \\
\hline Total & 100,0 & 100,0 & 100,0 & 100,0 & 100,0 & 100,0 \\
\hline
\end{tabular}

Fuente: Movimiento Natural de la Población. Media de los años 1995, 1996 y 1997. Elaboración propia. 
TABLA 8

Porcentaje de primíparas sobre total de mujeres en cada categoría de estado civil y grupo de edad

\begin{tabular}{lrrrrrr}
\hline Estado civil & $\begin{array}{c}\text { Menor } \\
\text { de } 20\end{array}$ & $20-24$ & $25-29$ & $30-34$ & $35-39$ & $\begin{array}{c}\text { Mayor } \\
\text { de 40 }\end{array}$ \\
\hline Casadas & 90,5 & 49,6 & 36,6 & 14,5 & 2,7 & 0,2 \\
\hline No casadas & 1,3 & 1,5 & 2,6 & 5,3 & 4,9 & 0,8 \\
\hline
\end{tabular}

Fuente: Elaboración propia con datos del Movimiento Natural de la Población (media de los años 1995, 1996 y 1997) y Encuesta de la Población Activa 1996.

El análisis de los intervalos protogénesicos no muestra pautas claras: las madres tardías no son mayoritariamente mujeres que esperan un tiempo prolongado tras el matrimonio para tener hijos, ni mujeres que se casan tardíamente y tienen a sus hijos inmediatamente después (Tabla 9).

\section{TABLA 9}

Años de matrimonio de las mujeres primíparas casadas según edad. Datos nacionales

\begin{tabular}{lccccccc}
\hline $\begin{array}{l}\text { Años de } \\
\text { casada }\end{array}$ & $\begin{array}{c}\text { Menor de } \\
\mathbf{2 0}\end{array}$ & $\mathbf{2 0 - 2 4}$ & $\mathbf{2 5 - 2 9}$ & $\mathbf{3 0 - 3 4}$ & $\mathbf{3 5 - 3 9}$ & $\begin{array}{c}\text { Mayor de } \\
\mathbf{4 0}\end{array}$ & Total \\
\hline Menos de 1 & $\mathbf{8 1 , 2}$ & 41,9 & 13,0 & 10,7 & 15,6 & 14,3 & 18,6 \\
\hline $\mathbf{1}$ & 12,7 & 27,6 & 23,4 & 18,9 & 19,2 & 15,2 & 22,0 \\
\hline 2 & 3,9 & 16,4 & 23,4 & 17,9 & 11,7 & 10,2 & 9,2 \\
\hline 3 & 1,7 & 8,2 & 17,3 & 14,6 & 7,9 & 6,8 & 14,0 \\
\hline 4 & 0,3 & 3,6 & 10,6 & 11,3 & 6,3 & 4,6 & 9,2 \\
\hline 5 y más & 0,2 & 2,4 & 12,2 & 26,4 & 39,3 & 48,8 & 17,0 \\
\hline Total & 100,0 & 100,0 & 100,0 & 100,0 & 100,0 & 100,0 & 100,0 \\
\hline
\end{tabular}

Fuente: Movimiento Natural de la Población. Media de los años 1995, 1996 y 1997. Elaboración propia.

Llama la atención la existencia de un porcentaje importante de madres muy tardías (de más de cuarenta años) que llevan más de cinco años casadas cuando tienen su primer parto, lo que puede deberse a problemas de infertilidad, ya que resulta difícil imaginar que se agoten hasta tal punto los límites biológicos.

\section{LA DIVERSIDAD REGIONAL EN ESPAÑA: MATERNIDAD TARDÍA... ¿ESTRATEGIA DE GÉNERO O MODELO TERRITORIAL DE FORMACIÓN DE FAMILIAS?}

La diversidad regional en España es muy grande cuando tenemos en cuenta la importancia cuantitativa de la maternidad tardía, tal y como se podía apreciar en la 
Tabla 2. Es sabido que existen variaciones notables en los niveles de fecundidad de las diecisiete Comunidades Autónomas que integran el Estado, variaciones que responden a una clara división norte-sur. Si consideramos el calendario reproductivo podemos ver que existe un área de maternidad tardía integrada por el País Vasco, Navarra, Aragón, Comunidad de Madrid y Rioja, así como Castilla-León y Cataluña. Las Comunidades con maternidad más temprana son Andalucía, Canarias, Extremadura, Murcia y Galicia. Hay, pues, pautas territoriales diferentes por lo que respecta al calendario de la maternidad -estando las diferencias netas en torno a los tres años, entre los valores máximos (País Vasco con 29,69) y mínimos (Ceuta y Melilla, con 26,57)- aunque estas diferencias no se ajustan exactamente a la división norte-sur que encontramos en relación a la fecundidad, ya que la cornisa atlántica presenta una maternidad bastante temprana (Mapa 1).

MAPA 1

Edades medias de las mujeres al primer parto por Comunidades Autónomas

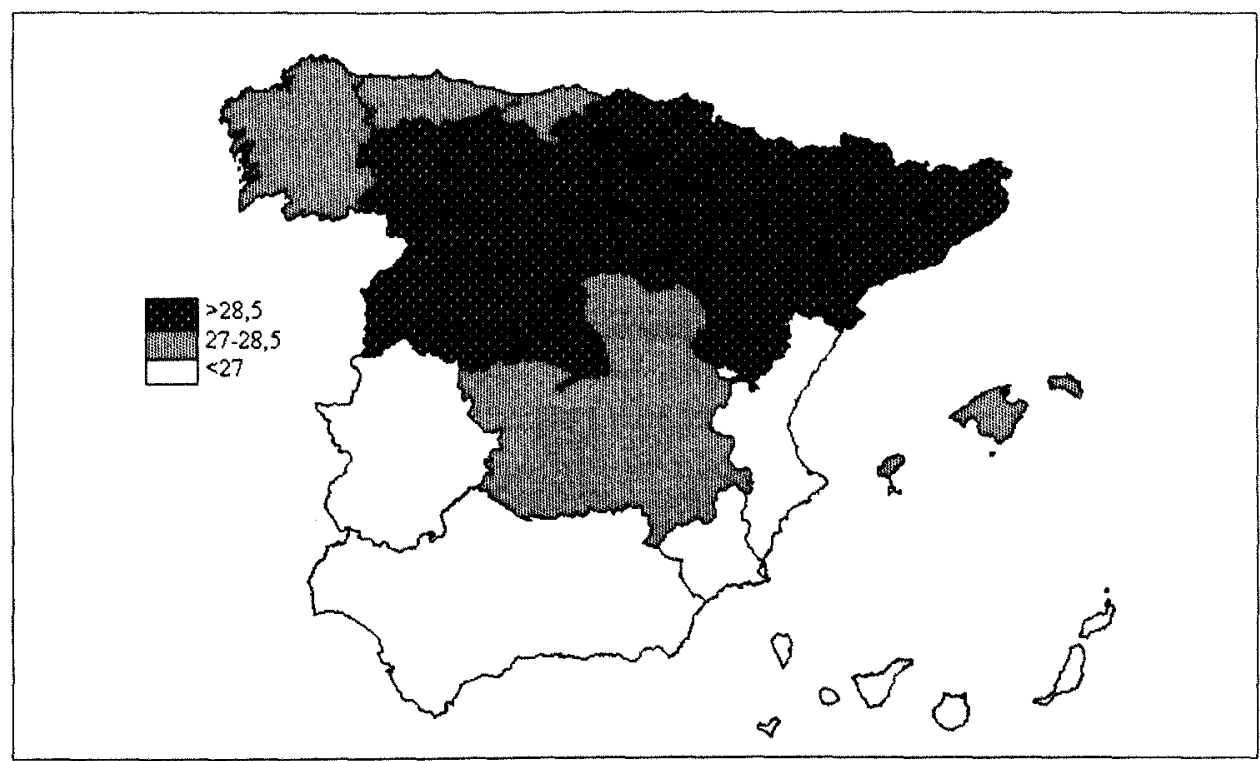

Cuando tenemos en cuenta esta diversidad surge automáticamente la pregunta de si la maternidad tardía responde y acompaña necesariamente a un determinado modelo de inserción laboral de la mujer (si es una estrategia de género, en definitiva) o si tiene que ver también con pautas culturales y territoriales relacionadas con ciertos modelos de formación de la familia que estimulan el retraso del matrimonio y de la llegada de los hijos. Si comparamos la edad media al primer parto de diferentes categorías de mujeres en diferentes Comunidades Autónomas, podemos comprobar si realmente existen «modelos» culturales de formación de la familia que van más allá de los condicionamientos y de las estrategias que acompañan a un determinado estatus laboral de la mujer. En el Gráfico 1 podemos comprobar que parecen existir modelos 
territoriales de formación de la familia, modelos que pesan más en el comportamiento femenino cuanto menor es el nivel de cualificación laboral de las mujeres. Las amas de casa vascas y canarias, por ejemplo, presentan una media de edad al primer parto netamente diferentes, como es diferentes la edad de las obreras y empleadas en comercio o servicios en estas mismas Comunidades. Las pautas en ambos territorios se acercan mucho más cuando consideramos a las trabajadoras cualificadas, y sobre todo a las profesionales y técnicas. Es como si al adquirir un elevado nivel profesional las mujeres se vieran impulsadas a seguir modelos de conducta uniformes, más allá de los patrones culturales en que se integren. En definitiva, a mayor nivel de formación y de cualificación profesional mayor homogeneidad observamos en el calendario reproductivo femenino, en los distintos territorios. La uniformidad de comportamientos que podría esperarse de una mayor uniformidad cultural, en un contexto de globalización y de mayor preeminencia de los valores postmaterialistas, está matizada por la existencia de elementos etnoterritoriales que muestran una fuerza considerable y actúan en aquellos sectores de la población con menor nivel sociocultural (y más impermeable, según la tesis del propio Inglehart) a estos nuevos valores.

GrÁFICO 1

Edad media de las mujeres al primer parto según condición laboral y Comunidades Autónomas

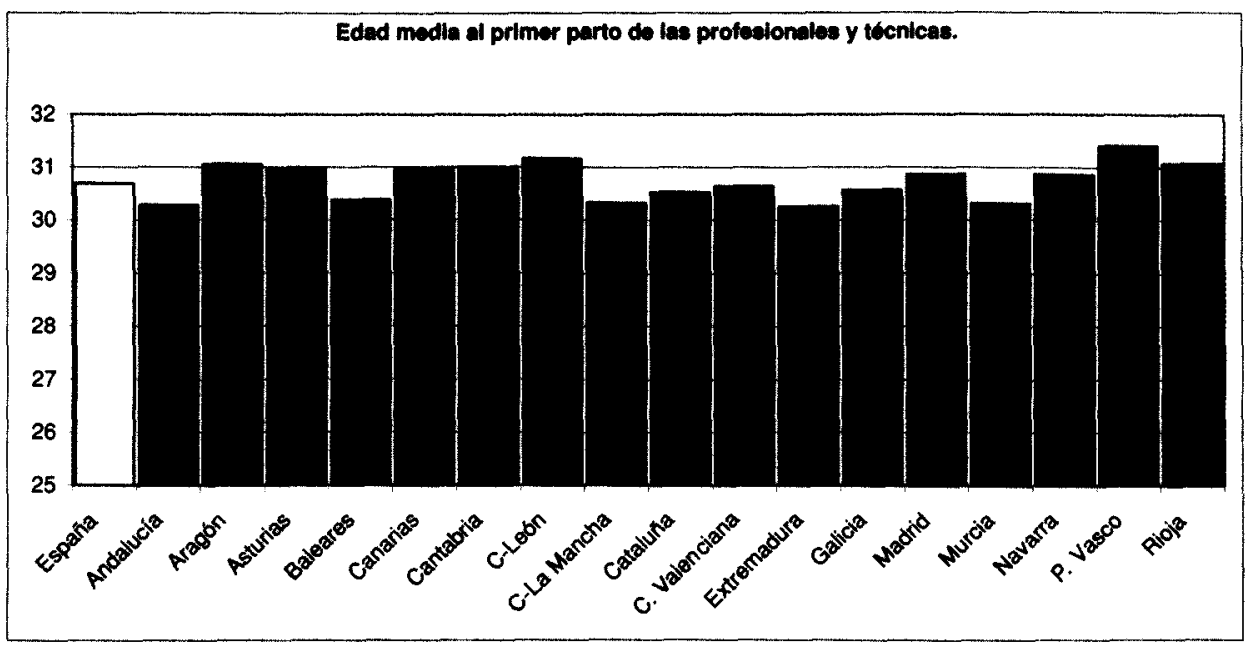



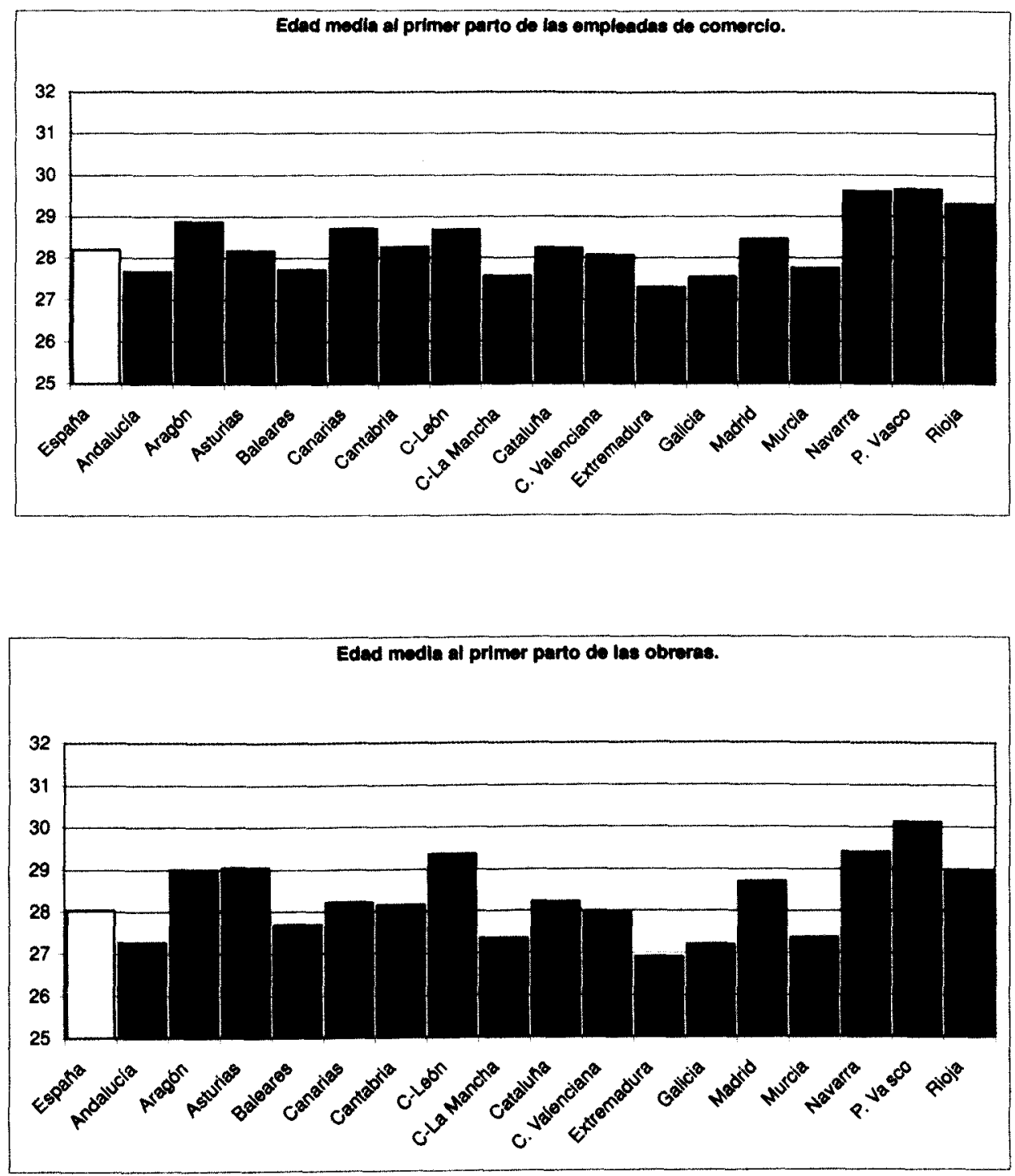

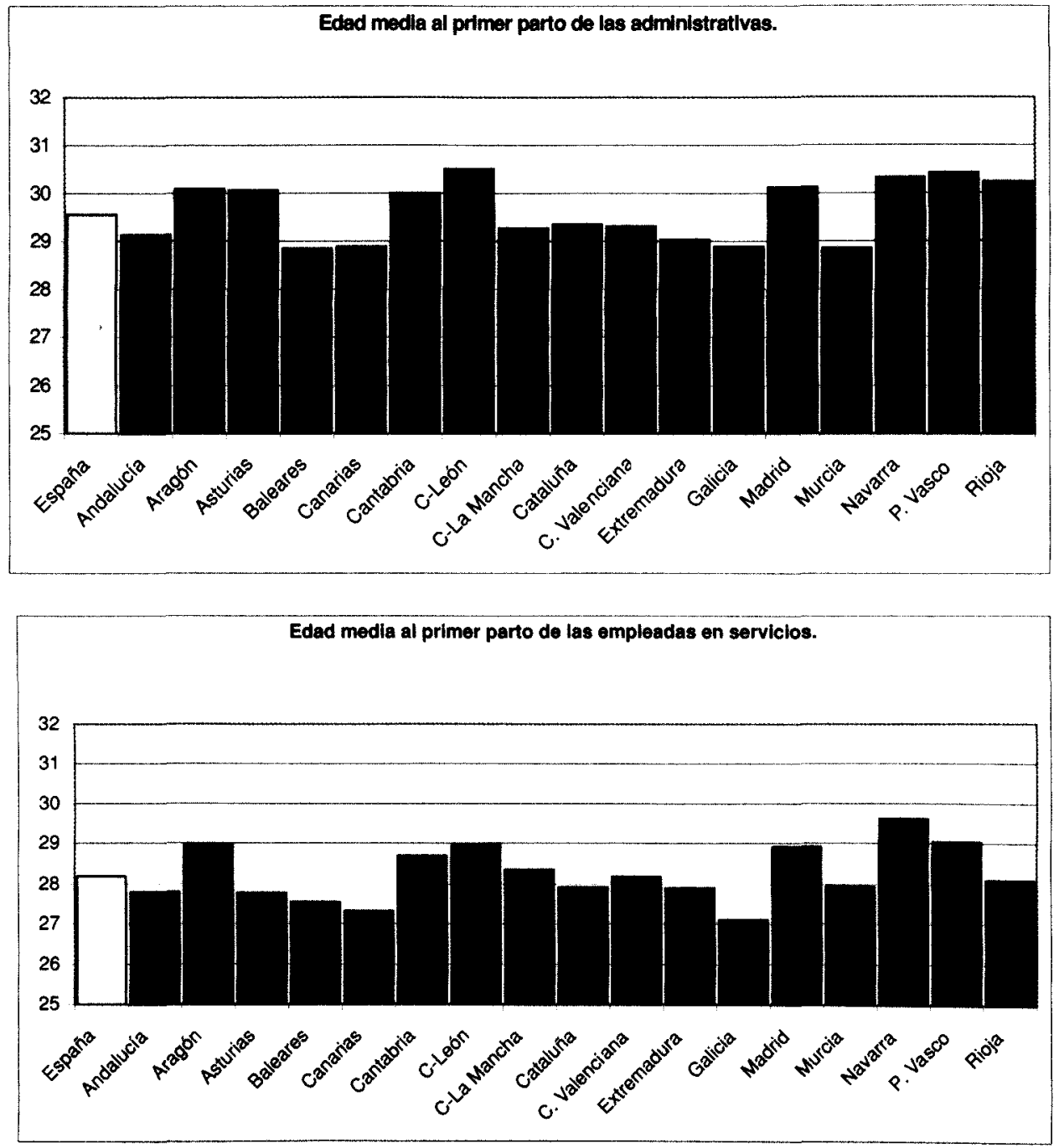


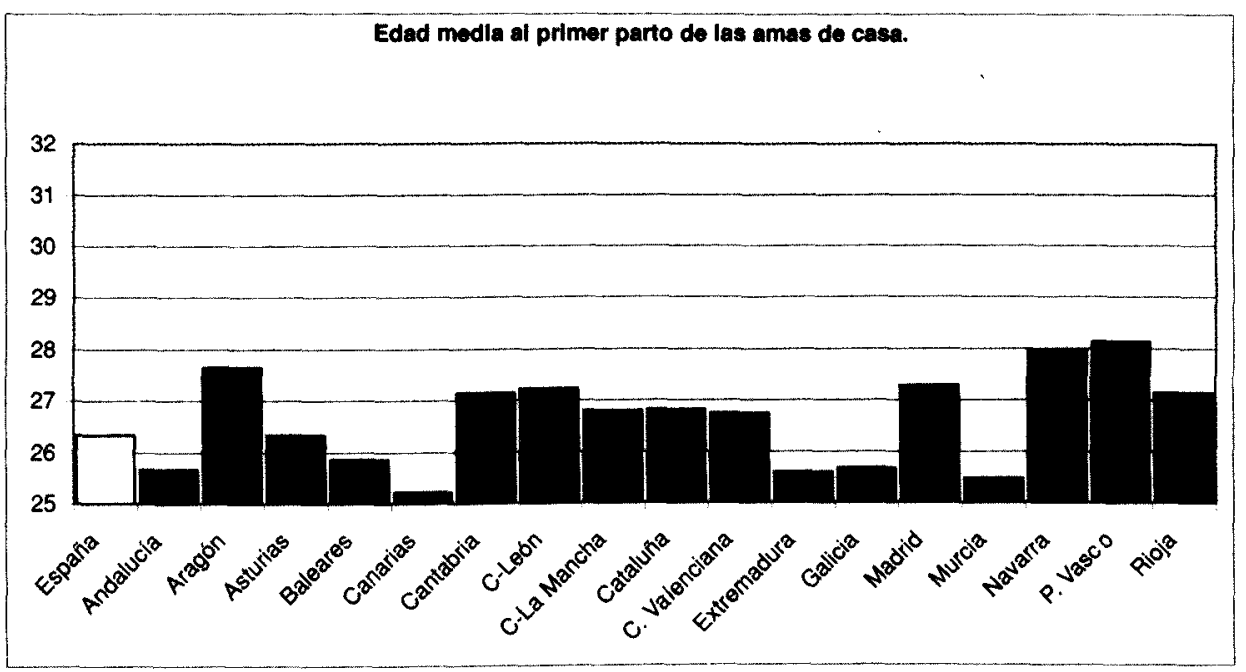

\section{LOS DISCURSOS EN TORNO A LA MATERNIDAD TARDÍA: ¿DÓNDE ESTÁ EL PROBLEMA?}

Lo primero que cabe destacar de los discursos recogidos entre madres y padres tardíos en la Comunidad Autónoma de Madrid es una valoración de la maternidad tardía como opción vital, que contrasta con los discursos negativos que normalmente encontramos en la literatura académica y en los mass-media. La maternidad tardía no es considerada negativamente, como algo extraño o producto de algún fracaso o fallo vital, sino la única conducta esperable en las mujeres que estudian y aspiran a tener una vida profesional. Tener hijos tarde es el resultado lógico de unas trayectorias vitales que se estructuran de una forma muy definida: finalización de los estudios, logro de una situación laboral estable y de un cierto capital de experiencia profesional, disfrute de un tiempo de consumo y realización personal (viajes...), consolidación de una situación económica que permita hacer frente a inversiones importantes como la compra de una vivienda, $y$, por fin, formación de una familia, con la llegada de los hijos.

Los hijos implican el fin de una fase vital caracterizada por la libertad y el hedonismo (el fin de la juventud propiamente dicha) y el comienzo de una situación de dependencia y de asunción de nuevas responsabilidades. Por eso nunca debe llegar antes de que la persona esté «preparada». Realmente, mientras en el discurso de las entrevistadas no existe una definición clara de lo que es maternidad tardía, si se sugiere una de lo que es maternidad temprana: una persona tiene hijos demasiado «pronto», independientemente de la edad que tenga, cuando no ha cerrado esa trayectoria de forma sosegada y natural. Aunque se reconocen ciertas ventajas en la maternidad temprana -mayor fortaleza física, una posible mayor cercanía en mentalidad a los hijos- éstas quedan anuladas por los inconvenientes que conlleva quebrar ese camino vital ideal. De ahí que «entre los 
treinta y los cuarenta sea la mejor edad», que la maternidad tardía implique serenidad, estabilidad, capacidad para asumir responsabilidades, tanto por parte de los padres como de las madres, y en definitiva un mayor disfrute de los hijos. Solo una sombra aparece ligada a la maternidad tardía y tiene que ver con los límites biológicos de la fertilidad: el aumento de la esterilidad, de los embarazos frustrados y del peligro de tener hijos con minusvalías físicas o psíquicas. La angustia con la que las mujeres se refieren a la ammiocentesis es una buena prueba de ello.

Por otra parte, aunque la maternidad tardía aparece como fruto de una elección libre y personal, como resultado de una determinada opción de las mujeres, también se vincula en los discursos de las entrevistadas a una especie de «fuerza» o «instinto» que hace que la gran mayoría de ellas «necesite» ser madre. La maternidad adquiere así, paradójicamente, la condición de algo natural para las mujeres, que las predispone a tener hijos, que les proporciona una relación privilegiada con ellos (en comparación con los padres) y que las ayuda a superar los inconvenientes de la crianza.

Maternidad y paternidad no son condiciones equivalentes, ya que la maternidad tiene mucho de instintivo, de inmediato, mientras que la paternidad es algo aprendido. El deseo de tener hijos está más definido entre las mujeres, mientras que a los hombres frecuentemente hay que «empujarles» o «convencerles» para dar ese paso. La relación de los hombres con el hecho de la maternidad y los hijos es indirecta, externa (los hombres lo ven y lo viven «desde fuera»). Esta relación, que hace de la maternidad algo fundamentalmente femenino, es contemplada por las mujeres con una mezcla de compasión (por lo que ellos pierden de protagonismo en la reproducción de la vida, y de relación privilegiada con los hijos) y de envidia (por las responsabilidades que de esta manera se evitan). Esta visión de la maternidad y la paternidad es compartida por las mujeres y los hombres entrevistados y parece ser un pilar fundamental en el mantenimiento de una división del trabajo según el género bastante tradicional, incluso entre personas con un nivel de formación y cualificación elevado.

La maternidad es descrita como una experiencia maravillosa, que llena, colma y cambia la vida, una vivencia que las mujeres no quieren «perderse». Pero al mismo tiempo las mujeres la perciben como algo absorbente, agobiante, que empobrece, resta individualidad y amenaza la vida profesional. El cuidado de los hijos, sobre todo en los primeros meses, provoca una sensación de aislamiento del mundo y de gran agobio.

La maternidad tardía como estrategia destinada a obtener una mayor estabilidad laboral y una posición de más fuerza en el mercado laboral cobra enorme sentido, ya que existe una conciencia clara de que los hijos amenazan el empleo y la promoción profesional de las mujeres. Todo lo que implica la maternidad, desde las molestias y complicaciones del embarazo, la baja maternal, el posible absentismo laboral o la disminución del tiempo y de la atención dedicada al trabajo, son elementos que contribuyen a hacer de las mujeres trabajadoras sospechosas de baja productividad, y por tanto prescindibles. Las mujeres intentan contrarrestar todas estas expectativas negativas aumentando su rendimiento e intentando cumplir al máximo en su trabajo, lo que en ocasiones provoca un gran 
agotamiento y estrés. A mayor nivel de cualificación, mayor es además la exigencia de continuo reciclaje, de dedicación al trabajo y de flexibilidad temporal y espacial, y mayor también la aspiración a desarrollar una carrera profesional. De ahí, que la maternidad sea un handicap importante en la vida profesional de las mujeres, en la medida en que la implicación en el cuidado y atención de los hijos siempre es mayor que la de los hombres.

Como no podría ser de otra forma, las dificultades para compatibilizar vida laboral y familiar aparecen patentes en el discurso de las madres tardías, incluso cuando éstas disponen de recursos formativos y económicos importantes. Las estrategias desplegadas para compatibilizar trabajo y familia tienen que ver más con la reducción del tiempo dedicado al trabajo (abandono temporal o definitivo, trabajo a tiempo parcial, etc) y la movilización de los recursos económicos (ayuda doméstica pagada, guarderías, etc.) que con una mayor corresponsabilidad en la pareja. A pesar de reconocer que los padres tardíos son mejores padres (de igual manera que las madres tardías son «mejores» madres -están más preparadas para afrontar la maternidad-), éstos no parecen implicarse excesivamente en el trabajo doméstico y en la atención a los hijos, siendo su papel siempre el de ayudante puntual de la mujer. La disponibilidad de ayuda familiar, sobre todo de las abuelas, cuando éstas están dispuestas y pueden cuidar de los niños, sigue apareciendo como un recurso importante, pero al que se intenta recurrir solo de forma esporádica.

Por todo ello, existe una clara conciencia de la soledad de las mujeres a la hora de compatibilizar vida familiar y laboral, ya que solo se cuenta en esta tarea con el propio esfuerzo y la ayuda ocasional de la familia. Las mujeres entrevistadas se muestran muy críticas con la falta de apoyo social a la maternidad y escandalizadas de que esta falta de apoyo se produzca al mismo tiempo que un general lamento por la baja natalidad. Las principales demandas se centran en una baja maternal más prolongada (idealmente de un año), mayor número de guarderías y más baratas, establecimiento de guarderías en los centros de trabajo y compensaciones económicas para las mujeres por reducción de jornada.

Las dificultades para compaginar hijos y trabajo se describen y afrontan desde un deseo de compatibilizar ambas esferas. El discurso dominante entre las mujeres entrevistadas es, en efecto, el de la «compatibilización». Desde este discurso se considera que el trabajo es algo irrenunciable, es el fruto de una conquista irreversible, conseguida por varias generaciones de mujeres que otorga independencia económica, derechos propios, una vida social y personal rica e individualidad (algo que no tienen las personas que viven en función de otras, como las amas de casa o «nuestras madres»). El trabajo, además, sea mejor o peor, es «lo tuyo», lo que se ha conseguido con mucho esfuerzo y se debe conservar. Ser madre y trabajadora es difícil porque ni la sociedad ni los hombres se implican y hoy por hoy las mujeres deben cubrir todos los frentes, pero merece la pena el esfuerzo de no renunciar a la vida profesional. Desde esta perspectiva se pretende que la maternidad sea un «paréntesis» en el que se ralentiza la vida profesional, pero en absoluto se interrumpe. Un «paréntesis» que incluso puede ser una experiencia positiva y enriquecedora, un cambio de actividad que no amenace al estatus laboral. Este proyecto ideal solo es realizable, sin embargo, para las funcionarias, de ahí 
que esta condición laboral siga contemplándose como la ideal para compatibilizar familia y trabajo. Algo que haría más real este "paréntesis" sería la prolongación de la baja maternal a un año (lo que evitaría el mal trago de dejar a los niños demasiado pequeños en la guardería) y la posibilidad de que los hombres tuvieran también una baja paternal que cubriera al menos las primeras y más difíciles semanas de adaptación al bebé.

Frente al discurso de la compatibilización, nos encontramos el discurso de la renuncia (al trabajo para cuidar a los hijos). Este discurso no se basa en lo positivo de la condición de ama de casa sino en la constatación de las enormes dificultades que supone la compatibilización y la aceptación resignada de la situación («de boquilla todos somos muy modernos, pero después....»). El discurso de la renuncia afirma que las mujeres que continúan trabajando cuando tienen hijos pierden dinero (ya que su sueldo se va en pagar guarderías o ayuda doméstica), pierden calidad de vida, por las prisas, los nervios y el estrés, y además cargan con sus propias responsabilidades a los abuelos y abuelas cuidadoras. La renuncia al trabajo, se argumenta por tanto, supone evitar todos esos males y asumir las propias responsabilidades, aunque para la mujer sea una decisión arriesgada perder su independencia económica. El discurso de la renuncia subraya que la vida del ama de casa no tiene por qué ser vacía y aburrida, ya que cuando el trabajo de cuidado y atención a la familia no llena todo el tiempo, hay una gran variedad de actividades culturales y recreativas a las que poder recurrir. En general este discurso es minoritario y en cierto sentido muy contradictorio, ya que frente a la señalada voluntariedad de la decisión de ser ama de casa se dejan entrever, al menos en el discurso de las entrevistadas, el peso de las tensiones y conflictos que se generan al compatibilizar vida familiar y laboral y las presiones de la pareja por mantener los roles de género tradicionales.

$\mathrm{La}$ indagación cualitativa viene a reafirmar los factores que están influyendo decisivamente en el retraso de la maternidad. El cambio de roles de la mujer, y sus aspiraciones a tener una vida profesional propia hacen de la maternidad tardía un resultado lógico de las fuertes inversiones en educación y una estrategia destinada a consolidar el estatus laboral para que la llegada de los hijos amenace lo menos posible esta posición. Son las mujeres profesionales las que más claramente materializan esta pauta. Por otro lado, vemos cómo las entrevistadas «colocan» a los hijos al final de un trayecto ideal que debe incluir necesariamente la consolidación de la situación económica y laboral de la pareja y una época de disfrute y realización personal previa a la asunción de responsabilidades familiares. Tras esta pauta aparece de nuevo el modelo de familia que responde por una parte a ese «pacto privado para la felicidad», al que se refería Roussel, y también propio de la Europa del sur en el que hay que tener «proyectos de vida muy claros» como señalaba Livi Bacci.

El deseo de ser madre aparece en el discurso de las entrevistadas como algo que va más allá de lo racional y que hace de la maternidad algo instintivo, natural para las mujeres, a pesar de que, paradójicamente, tener hijos sea una opción personal, libre y muy meditada. La maternidad es una experiencia que puede posponerse pero no «perderse». Esta «necesidad» de convertirse en madres que muestran las mujeres, el carácter instintivo e inmediato que tiene la maternidad para ellas, fren- 
te a una paternidad mucho más aprendida, racionalizada y mediata, convierte a los hijos en un asunto femenino más allá de lo que sería deseable en la gestión de lo cotidiano. De hecho, este deseo «natural» de las mujeres por los hijos, lleva a que incluso entre los colectivos con un nivel formativo elevado, aparezca una división del trabajo doméstico entre hombres y mujeres altamente tradicional.

En un contexto social en el que el recurso a la ayuda de las mujeres de la familia (sobre todo las abuelas) se hace más y más complicado, y en el que la corresponsabilidad entre hombres y mujeres es todavía un deseo, las dificultades de las madres trabajadoras aumentan considerablemente cuando no existe un apoyo social que supla en cierta mediad las carencias anteriores. Las mujeres entrevistadas tienen muy claro que ese apoyo no existe o es insuficiente, y probablemente lo sepan también las mujeres que tienen el proyecto de ser madres, elaborando sus estrategias de acuerdo con estas carencias y posponiendo la llegada de los hijos hasta que los propios recursos sean suficientes para hacer frente al reto de compatibilizar vida laboral y familiar. Mientras tanto, las mujeres sueñan con otros escenarios en los que bajas maternales más prolongadas, guarderías más numerosas y baratas, apoyo económico y protección legal para las madres que trabajan contribuyan a hacer de la maternidad no una amenaza sino ese enriquecedor «paréntesis» en la vida profesional.

\section{BIBLIOGRAFÍA}

ALBERDI, I. (1999): La nueva familia española, Madrid: Taurus.

BECK, U. y BECK-GERNSHEIM, E. (2001): El normal caos del amor. Las nuevas formas de relación amorosa. Barcelona, Paidos.

Bosveld, W. y KuIsTten, A. (1995): «Delayed childbearing: generational change in life course patterns of fertility», Paper prepared for the Second ESA Conference of Sociology, European societies: fussion or fission, Working Group 5.6. «Generational change in postwar Europe», Budapest, August-September, 1995.

CASTRO, T. (1992): «Delayed childbearing in contemporary Spain: trends and differentials» en European Journal of Population, 8 (1992), pp. 217-246.

- (1999): «Pautas recientes en la formación de pareja» en Revista Internacional de Sociología, n. ${ }^{\circ} 23$, pp. 61-94.

Delgado, M. y CASTro, T. (1998): Encuesta de Fecundidad y Familia 1995 Centro de Investigaciones Sociológicas, Serie Opiniones y Actitudes, $n .^{\circ} 20$.

DIEZ NiCOLÁs, J. (2001): «Causas y consecuencias del reciente descenso de la fecundidad en España», en Demografía y Cambio Social, Comunidad de Madrid, Consejería de Servicios Sociales.

Esping-Andersen, G. (1990): The Three Worlds of Welfare Capitalism. Oxford, Polity Press.

GARRIDO, L. (1992): Las dos biografias de la mujer en España, Madrid: Instituto de la Mujer, Ministerio de Asuntos Sociales.

- (1996): «La revolución reproductiva» en Castaño, C. y Palacios, S., Salud, dinero y amor, Alianza Editorial. 205-238.

GidDENs, A. (1995): La transformación de la intimidad. Madrid, Cátedra.

Guillén, A. (1997): «Regímenes de bienestar y roles familiares. Un análisis del caso español» en Papers, 53, pp. 45-63. 
Iglesias de UsSEL, J. y MeIL, G. (2001): La política familiar en España. Barcelona, Ariel. MeIL, G. (1999): La postmodernización de la familia española. Madrid, Acento Editorial. ReHER, D.S. (1996): La familia en España. Pasado y presente. Madrid, Alianza Universidad.

REQuenA, M. (1997): «Sobre el calendario reproductivo de las mujeres españolas» en Reis, 79/97, pp. 43-79.

- (2002): «Formación de la pareja y fecundidad: una simulación con datos de la Comunidad de Madrid» en Empiria, 5.

ROUSSEL, L. (1992): «La famille en Europe occidentale: divergences et convergences» en Population, Vol. 47, N. 1, Jan-Feb 1992, pp. 133-152.

SARRIBLE, G. (1995): «Maternidad e infecundidad: Más madres, menos hijos» en Revista Internacional de Sociología, Tercera Época, n. 11, Mayo-Agosto 1995, pp. 115-137. - (1997): «Reproducción e imagen de la mujer. Crítica de género en demografía» en Papers, 53, 1997, pp. 11-24.

Toвío, C. (2001a): «La contradicción familia-empleo y las estrategias de las madres trabajadoras» en Demografía y Cambio Social, Comunidad de Madrid, Consejería de Servicios Sociales.

- (2001b): «Marriage, cohabitation and the residencial independence of young people in Spain», en International Journal of Law, Policy and the Family, 15, 68-87. 